\title{
AN EXTREMAL LENGTH PROBLEM ON A BORDERED RIEMANN SURFACE ${ }^{1}$ )
}

\author{
BY \\ JEFFREY CLAYTON WIENER
}

\begin{abstract}
Partition the contours of a compact bordered Riemann surface $R^{\prime}$ into four disjoint closed sets $\alpha_{0}, \alpha_{1}, \alpha_{2}$ and $\gamma$ with $\alpha_{0}$ and $\alpha_{1}$ nonempty. Let $F$ denote the family of all locally rectifiable 1-chains in $R^{\prime}-\gamma$ which join $\alpha_{0}$ to $\alpha_{1}$. The extremal length problem on $R^{\prime}$ considers the existence of a real-valued harmonic function $u$ on $R^{\prime}$ which is 0 on $\alpha_{0}, 1$ on $\alpha_{1}$, a constant on each component $v_{k}$ of $\alpha_{2}$ with $\int v_{k}{ }^{*} d u=0$ and ${ }^{*} d u=0$ along $\gamma$ such that the extremal length of $F$ is equal to the reciprocal of the Dirichlet integral of $u$, that is, $\lambda(F)=D_{R}(u)^{-1}$.

Let $\bar{R}$ denote a bordered Riemann surface with a finite number of boundary components and $S$ a compactification of $\bar{R}$ with the property that $\partial \bar{R} \subset S$. We consider the extremal length problem on $\bar{R}$ (as a subset of $S$ ) when $\alpha_{0}, \alpha_{1}$, and $\alpha_{2}$ are relatively closed subarcs of $\partial \bar{R}$ and when $\alpha_{0}, \alpha_{1}$ and $\alpha_{2}$ are closed subsets of $\partial S=(S-\bar{R}) \cup \partial \bar{R}$.
\end{abstract}

Introduction. A. Marden and B. Rodin [5, pp. 237-269] extended the extremal length problem on a compact bordered Riemann surface by considering the Kerékjártó-Stoilöw compactification $R^{*}$ of an open Riemann surface $R$. They partitioned the ideal boundary $\beta=R^{*}-R$ of $R$ into four disjoint sets $\alpha_{0}, \alpha_{1}, \alpha_{2}$ and $\gamma$ with $\alpha_{0}$ and $\alpha_{1}$ nonempty and $\alpha_{0}, \alpha_{1}$ and $\alpha_{0} \cup \alpha_{1} \cup$ $\alpha_{2}$ closed in $R^{*}$. They showed that if the extremal length of the family $F^{*}$ of all locally rectifiable 1-chains in $R^{*}-\gamma$ which join $\alpha_{0}$ to $\alpha_{1}$ is finite, then there exists a real-valued harmonic function $u$ on $R$ which is 0 on $\alpha_{0}, 1$ on $\alpha_{1}$, a constant on each component $\tau_{k}$ of $\alpha_{2}$ with the constant chosen so that $\int_{\tau_{k}}{ }^{*} d u=0$ and ${ }^{*} d u=0$ along $\gamma$ (in the sense of limits along curves tending to the ideal boundary) such that $\lambda\left(F^{*}\right)=D_{R}(u)^{-1}$.

Let $\vec{R}$ denote a bordered Riemann surface with a finite number of boundary components and $S$ a compactification of $\bar{R}$ with the property that $\partial \bar{R} \subset S$ (see Theorem 4). Set $\beta=S-\bar{R}$ and $\partial S=\beta \cup \partial \vec{R}$. Partition $\partial \vec{R}$ into the four

Received by the editors December 4, 1973 and, in revised form, April 19, 1974.

AMS (MOS) subject classifications (1970). Primary 31 A05, 31 A15.

Key words and phrases. Extremal length, harmonic function, Kerékjártó-Stoilöw compactification, canonical exhaustion, double of a surface, regular exhaustion.

(1) The results in this paper were part of the author's doctoral dissertation which was written at Washington University, St. Louis, under the direction of Professor James A. Jenkins. 
sets $\alpha_{0}, \alpha_{1}, \alpha_{2}$ and $\gamma$, where $\alpha_{0}$ and $\alpha_{1}$ are nonempty, $\alpha_{0}, \alpha_{1}$ and $\alpha_{2}$ are each composed of a finite number of relatively closed subarcs of $\partial \bar{R}$ and closed contours of $\bar{R}$, the closures on $S$ of $\alpha_{0}, \alpha_{1}$ and $\alpha_{2}$ are pairwise disjoint and $\gamma=\partial S-\left(\alpha_{0} \cup \alpha_{1} \cup \alpha_{2}\right)$. Let $F$ denote the family of all locally rectifiable 1chains in $S-\gamma$ which join $\alpha_{0}$ to $\alpha_{1}$. Theorem 5 solves the extremal length problem on $\bar{R}$.

Theorems 6 and 7 consider the extremal length problem on $\bar{R}$ when $\alpha_{0}$, $\alpha_{1}$ and $\alpha_{2}$ are closed subsets of $\partial S$.

REMARK 1. Suppose that the closure on $S$ of each component of $\alpha_{0} U$ $\alpha_{1} \cup \alpha_{2}$ is a closed subset of $\vec{R}$. By considering the double of $\bar{R}$ across $\gamma$ and using [5] we can solve the extremal length problem on $\bar{R}$. For this reason throughout this paper we shall assume that this is not the case.

REMARK 2. Suppose that $\bar{R}$ is any bordered Riemann surface and that the sets $\alpha_{0}, \alpha_{1}$ and $\alpha_{2}$ are chosen so that they intersect a finite number of contours of $\vec{R}$. By doubling $\vec{R}$ across the remaining contours we obtain a bordered Riemann surface with a finite number of boundary components. Therefore, since we will only consider the case when $\alpha_{0} \cup \alpha_{1} \cup \alpha_{2}$ intersects a finite number of contours of $\bar{R}$, it suffices to consider the extremal length problem on a bordered Riemann surface with a finite number of boundary components.

1. Preliminaries. Throughout this paper we tacitly assume that all harmonic functions are real-valued and that all curves and chains are locally rectifiable.

Let $\bar{R}$ denote a bordered Riemann surface with a finite number of boundary components and border $\partial \bar{R}$. Each component of $\partial \bar{R}$, called a contour of $\bar{R}$, can be classified as either type I or type II, where a contour of $\bar{R}$ is said to be of type I if it is the homeomorphic image of a circle or type II if it is the homeomorphic image of the open unit interval.

Partition the contours of $\bar{R}$ into four sets $\alpha_{0}, \alpha_{1}, \alpha_{2}$ and $\gamma$, where $\alpha_{0}$ and $\alpha_{1}$ are nonempty. Define the fanily $F$ of dll locally rectifiable chains in $\bar{R}-\gamma$ joining $\alpha_{0}$ to $\alpha_{1}$ by $C \in F$ if and only if $C$ is a continuous map from a union of closed intervals $\left[a_{0}, a_{1}\right] \cup\left[a_{2}, a_{3}\right] \cup \cdots \cup\left[a_{n-1}, a_{n}\right]$ into $\vec{R}-\gamma$ with $C\left(a_{0}\right) \in \alpha_{0}, C\left(a_{n}\right) \in \alpha_{1}$ and, for odd $j<n, C\left(a_{j}\right)$ and $C\left(a_{j+1}\right)$ belong to the same component of $\alpha_{2}$.

Traditionally the elements of $F$ are called curves.

Let $\rho|d z|$ denote a metric (linear density) on $\bar{R}$ and $H$ the family of all metrics $\rho|d z|$ on $\bar{R}$ such that $L(F, \rho|d z|)=\inf _{c \in F} \int_{c} \rho|d z|$ and $A(\rho|d z|, \bar{R})=\iint_{\bar{R}} \rho^{2} d x d y$ are defined and not simultaneously 0 or $+\infty$. The least upper bound $\lambda(F)$ of $L^{2}(F, \rho|d z|) / A(\rho|d z|, \bar{R})$ over all $\rho|d z| \in H$ is called the extremal length of $F$. It is a conformal invariant ([2], [3], [4], [8] or [10]). 
If $F^{*}$ denotes the family of all curves on $\bar{R}$ which separate $\alpha_{0}$ and $\alpha_{1}$, then $\lambda\left(F^{*}\right)$ is called the conjugate extremal length of $F$. For the results in this paper $\lambda\left(F^{*}\right)=\lambda(F)^{-1}$ (see $[8$, pp. 124, 128]).

Let $u$ denote a harmonic function on $\bar{R}$. Then ${ }^{*} d u=-u_{y} d x+u_{x} d y$ is a linear differential on $\bar{R}$. We say that $* d u=0$ along a differentiable curve $z(t)=x(t)+i y(t)$ if $-u_{y} x_{t}+u_{x} y_{t}=0$ for all $t$ and that the flux of $u$ across $z(t)$ is the integral of $* d u$ over $z(t)$.

The following theorem will be needed (see $[2$, p. 147] or $[8$, p. 36]).

THEOREM 1. Let $G$ be a subdomain of a Riemann surface $R$, and suppose that a harmonic function $u_{\Omega}$, defined in $G$, is assigned to every sufficiently large regular subdomain $\Omega$ of $R$. Then, if $D_{G}\left(u_{\Omega}-u_{\Omega^{\prime}}\right) \rightarrow 0$ as $\Omega, \Omega^{\prime} \rightarrow R$, there exists a harmonic function $u$ in $G$ with the following properties:

(i) $D_{G}\left(u_{\Omega}-u\right) \rightarrow 0$ as $\Omega \rightarrow R$,

(ii) $D_{G}\left(u_{\Omega}\right) \rightarrow D_{G}(u)$ as $\Omega \rightarrow R$,

(iii) $u_{\Omega}(z)-u_{\Omega}\left(z_{0}\right) \rightarrow u(z)$ as $\Omega \rightarrow R$.

In (iii), $z_{0}$ is an arbitrary point in $G$, fixed in advance, and the convergence is uniform on every compact subset of $G$.

2. Known results. Partition the contours of a compact bordered Riemann surface $T$ into four distinct sets $\alpha_{0}, \alpha_{1}, \alpha_{2}$ and $\gamma$ with $\alpha_{0}$ and $\alpha_{1}$ nonempty. Further, if $\alpha_{2}$ is nonempty, partition its contours into a finite number of distinct sets $\left\{\tau_{k}\right\}$.

Let $u$ denote the unique harmonic function on $T$, see [2, p. 157], which is 0 on $\alpha_{0}, 1$ on $\alpha_{1}$, a constant on each part $\tau_{k}$ of $\alpha_{2}$ with the constant chosen so that the flux of $u$ across $\tau_{k}$ is zero and $* d u=0$ along $\gamma$. Let $F$ denote the family of all curves in $T-\gamma$ which join $\alpha_{0}$ to $\alpha_{1}$. The following result is known [5, pp. 250-251].

\section{THEOREM 2. $\lambda(F)=D_{T}(u)^{-1}$.}

Marden and Rodin [5] used the Kerékjártó-Stoilöw compactification of a Riemann surface to extend this result.

Let $R$ be a Riemann surface and $R^{*}$ its Kerékjártó-Stoilöw compactification [2, pp. 81-87]. Partition the ideal boundary of $R, R^{*}-R$, into four disjoint sets $\alpha_{0}, \alpha_{1}, \alpha_{2}$ and $\gamma$ with $\alpha_{0}$ and $\alpha_{1}$ nonempty and $\alpha_{0}, \alpha_{1}$ and $\alpha_{0} \cup \alpha_{1} \cup \alpha_{2}$ closed in $R^{*}$. Let $F^{*}$ denote the family of curves in $R^{*}-\gamma$ which join $\alpha_{0}$ to $\alpha_{1}$. Marden and Rodin proved that if $\lambda\left(F^{*}\right)<\infty$, then there is a harmonic function $u$ on $R$ which is 0 on $\alpha_{0}, 1$ on $\alpha_{1}$, a constant on each component $\tau_{k}$ of $\alpha_{2}$ with the flux of $u$ across $\tau_{k}$ equal to 0 and ${ }^{*} d u=0$ along $\gamma$ (in thesense of limits along curves tending to the ideal boundary) so that 
THEOREM 3. $\lambda\left(F^{*}\right)=D_{R}(u)^{-1}$.

3. The compactification $S$. The compactification of $\bar{R}$ that we will use to generalize Theorem 3 is obtained by modifying Ahlfors and Sario's construction of the Kerékjártó-Stoilöw compactification of an open Riemann surface [2, pp. 82-87].

Let $T$ be a topological space. A set $\beta \subset T$ is said to be nonseparating on $T$ if every domain $G \subset T$ remains connected after removal of all points in $G \cap \beta$.

For convenience denote by $Q$, with or without subscripts, any domain on $\bar{R}$ which is not relatively compact, but has compact boundary. Note that $Q$ is never void.

DEFINITION. An ideal boundary component of $\bar{R}$ is a nonvoid collection $q$ of sets $Q$ which satisfy the following conditions: if $Q_{0} \in q$ and $Q \supset Q_{0}$, then $Q \in q$; if $Q_{1}, Q_{2} \in q$ there exists a $Q_{3} \in q$ with $Q_{3} \subset Q_{1} \cap Q_{2}$; the intersection of all closures of $Q, Q \in q$, is empty.

The proof of the following theorem is analogous to that of Ahlfors and Sario [2, pp. 82-87] and shall be omitted.

THEOREM 4. There exists a unique compactification $S$ of $\vec{R}$ such that $S$ is a locally connected Hausdorff space, $\beta=S-\bar{R}$ is totally disconnected and $\beta$ is nonseparating on $S$.

Note that $\partial \vec{R}$ is a subset of $S$.

4. Pseudosymmetric exhaustion. In order to consider the possibility that $\alpha_{0} \cup \alpha_{1} \cup \alpha_{2}$ contains a component whose closure on $S$ contains a point of $S-\bar{R}$ it will be necessary to construct a "nearly canonical" exhaustion $\left\{R_{n}\right\}$ of $\bar{R}$ such that $R_{1}$ intersects each contour of $\vec{R}$.

Let $T$ denote the union of all type II contours of $\bar{R}$. Suppose that $T$ is nonempty. We modify Ahlfors and Sario's proof of the existence of an exhaustion of an open Riemann surface [2, pp. 144-145] to construct a "nearly canonical" exhaustion of $\bar{R}$.

Cover $\bar{R}$ by a countable number of parameter discs and parameter halfdiscs $V_{0 n}, V_{0 n}^{\prime}$, where $V_{0 n} \subset \bar{R}-T$ and $V_{0 n}^{\prime} \cap T \neq 0$. Without loss of generality assume that each $V_{0 j}^{\prime}$ intersects only one type II contour of $\vec{R}$, say $T_{j}$, and that $T_{1}, \cdots, T_{q}$ are the $q$ distinct components of $T$.

Let $\Gamma$ be a simple arc in $\bar{R}-\partial \bar{R}$ with $\Gamma \cap V_{0 j}^{\prime} \neq 0$ for $j=1, \cdots, q$. Assume that $V_{01}, \cdots, V_{0 m}, V_{01}^{\prime}, \cdots, V_{0 q}^{\prime}$ cover $\Gamma$ and pass to the double $D(\bar{R})$ of $\bar{R}$ at $T$.

Let $\mathrm{Cl}$ denote closure on $D(\bar{R})$. Following Ahlfors and Sario we construct a regular exhaustion $\left\{\mathrm{Cl} W_{k}^{*}\right\}$ of $D(\bar{R})$ such that each component $H_{k j}$ of $\mathrm{Cl} W_{k+1}^{*}-W_{k}^{*}$ is a compact bordered Riemann surface with a finite number of 
analytic Jordan boundary curves $B_{1}, \cdots, B_{r}$ on the boundary of $\mathrm{Cl} W_{k}^{*}$ and certain others on the boundary of $\mathrm{Cl} W_{k+1}^{*}$.

For $m=1,2, \cdots, r-1$ join $B_{m}$ and $B_{m+1}$ by two distinct arcs bounding a narrow strip $s_{m}$ so that $\mathrm{Cl} s_{m} \cap \mathrm{Cl} s_{j}=0$ for $m \neq j$. Deleting Us $s_{m}$ from $H_{k j}$ we obtain a compact bordered Riemann surface $H_{k j}^{\prime}$ bounded by a piecewise analytic Jordan curve $K_{j}$ and the boundary curves of $H_{k j}$ in the boundary of $\mathrm{Cl} W_{k+1}^{*}$. There is a Jordan curve $J_{k j}$ in $H_{k j}^{\prime}$ bounding with $K_{j}$ a doubly-connected domain in $H_{k j}$ [4, Lemma 8.1]. There is a Jordan curve $\Gamma_{k j}$ in this domain which separates the boundary components of the domain. For each $j$ add the subdomain of $H_{k j}$ bounded by $\Gamma_{k j}$ and $B_{1}, \cdots, B_{m}$ as well as these curves to $\mathrm{Cl} W_{k}^{*}$ to obtain a compact bordered Riemann surface $W_{k}$. The exhaustion $\left\{W_{k}\right\}$ is canonical.

Set $R_{k}=W_{k} \cap \bar{R}$. Then $\left\{R_{n}\right\}$ is a sequence of compact bordered Riemann surfaces on $\bar{R}$ with the following properties:

(i) $\cup R_{n}=\bar{R}, R_{n} \subset R_{n+1} \subset \vec{R}, \partial \vec{R}-T \subset \partial R_{n}$, and $\partial \vec{R} \cap t \neq 0$ for each component $t$ of $T$;

(ii) $\partial R_{n}$ is composed of analytic Jordan curves, open analytic Jordan arcs in $\bar{R}-\partial \vec{R}$ with end points on $\partial \vec{R}$ and subarcs of components of $T$;

(iii) each component of $\bar{R}-R_{n}$ is noncompact and its boundary consists of a single open analytic arc in $\bar{R}-\partial \bar{R}$ with end points on $\partial \bar{R}$;

(iv) $\partial R_{n}$ and $T$ are orthogonal at their points of intersection. of $\bar{R}$.

The exhaustion $\left\{R_{n}\right\}$ of $\vec{R}$ will be called a pseudosymmetric exhaustion

5. First result. We are ready to prove the following theorem.

THEOREM 5. Let $S$ denote the compactification of $\bar{R}$ defined above. Suppose that $\alpha_{0}, \alpha_{1}$ and $\alpha_{2}$ are subsets of $\partial \bar{R}$ which satisfy the following conditions:

(i) $\alpha_{0}$ and $\alpha_{1}$ are nonempty;

(ii) $\alpha_{0}, \alpha_{1}$ and $\alpha_{2}$ are composed of a finite number of relatively closed arcs and full contours of $\vec{R}$;

(iii) $\alpha_{0}, \alpha_{1}$ and $\alpha_{2}$ have pairwise disjoint closures on $S$.

Set $\beta=S-\bar{R}, \gamma=\beta \cup\left(\partial \vec{R}-\left(\alpha_{0} \cup \alpha_{1} \cup \alpha_{2}\right)\right)$ and denote by $F$ the family of all curves in $\vec{R}-\gamma$ which join $\alpha_{0}$ to $\alpha_{1}$. There exists a harmonic function $u$ on $\bar{R}$ which is 0 on $\alpha_{0}, 1$ on $\alpha_{1}$, a constant on each component $\tau_{k}$ of $\alpha_{2}$ with the constant chosen so that the flux of $u$ across $\tau_{k}$ is 0 and ${ }^{*} d u=0$ along $\gamma$ such that $0<\lambda(F)=D_{\bar{R}}(u)^{-1}<\infty$.

A precise meaning for ${ }^{*} d u=0$ along $\gamma \cap \partial \bar{R}$ exists. On $\gamma-\partial \bar{R}$ the sense is of a limit along curves tending to each component of $\gamma-\partial \vec{R}$. 
For the remainder of this paper $\mathrm{Cl}$ will represent the closure on $S$.

PROOF. Let $\left\{R_{n}\right\}$ denote a pseudosymmetric exhaustion of $\vec{R}$ and $\delta$ a component of $\alpha_{0} \cup \alpha_{1} \cup \alpha_{2}$. If $\mathrm{Cl} \delta \subset \partial \vec{R}$, assume that $\delta \subset \partial R_{1}$. If not, let $\sigma$ denote the type II contour of $\bar{R}$ which intersects $\delta$. If $\sigma-\delta \neq 0$, let $f$ denote a homeomorphism of $\sigma$ onto $\{t: 0<t<1\}$. There is a constant $b(0<b<1)$ such that $f^{-1}(\{t: b \leqslant t<1\})=\delta \cap \sigma$ or $f^{-1}(\{t: 0<t \leqslant b\})$ $=\delta \cap \sigma$. Set $\epsilon=\min (b, 1 / 2)$. Assume that $f^{-1}(\{t: b-\epsilon<t<b+\epsilon\}) \subset \partial R_{1}$.

Set $\alpha_{j n}=\alpha_{j} \cap \partial R_{n}(j=0,1$ and 2$)$ and $\gamma_{n}=\partial R_{n}-\left(\alpha_{0 n} \cup \alpha_{1 n} \cup \alpha_{2 n}\right)$. For each $n$, let $F_{n}$ denote the family of all curves in $R_{n}-\gamma_{n}$ which join $\alpha_{0 n}$ to $\alpha_{1 n}$ and let $u_{n}$ denote the harmonic function on $R_{n}$ satisfying Theorem 2. Since $F_{n} \subset F_{n+1}$ and $F=\bigcup F_{n}, \lambda(F)=\lim \lambda\left(F_{n}\right)$ and $\lambda\left(F_{n+1}\right) \leqslant \lambda\left(F_{n}\right)=$ $D_{R_{n}}\left(u_{n}\right)^{-1}<\infty$. Thus, $0 \leqslant \lambda(F)=\lim D_{R_{n}}\left(u_{n}\right)^{-1}<\infty$.

There exists a finite collection of pairwise disjoint open analytic Jordan arcs $\left\{J_{j}\right\}_{j=1}^{A}$ in $\bar{R}-\partial \vec{R}$ with end points on $\partial \vec{R} \cap \gamma$ and analytic Jordan curves $\left\{J_{j}\right\}_{j=A+1}^{B}$ in $\bar{R}-\partial \bar{R}$ which, when taken together, separate $\alpha_{0} \cup \alpha_{2}$ and $\alpha_{1}$. Suppose that $J_{j}(j=1, \cdots, B)$ separates the subset $\alpha_{1}(j)$ of $\alpha_{1}$ and $\alpha_{0} U$ $\alpha_{2} \cup\left(\alpha_{1}-\alpha_{1}(j)\right)$.

For $j=1, \cdots, A$ let $p_{1 j}$ and $p_{2 j}$ denote the end points of $J_{j}$ on $\partial \bar{R} \cap \gamma$. Take two open connected sets $U_{1 j}$ and $U_{2 j}$ on $\partial \vec{R}$ (relative to $\partial \vec{R}$ ) with $p_{1 j} \in U_{1 j} \subset \partial \bar{R} \cap \gamma$ and $p_{2 j} \in U_{2 j} \subset \partial \bar{R} \cap \gamma$. There exists an open analytic Jordan arc $L_{j}$ in $\vec{R}-\partial \bar{R}$ with end points $q_{1 j}$ on $U_{1 j}-\left\{p_{1 j}\right\}$ and $q_{2 j}$ on $U_{2 j}-\left\{p_{2 j}\right\}$ so that $L_{j}$ separates $\alpha_{1}(j)$ and $J_{j}$ and so that $J_{j}$, the arc on $U_{1 j}$ joining $p_{1 j}$ and $q_{1 j}$, the arc on $U_{2 j}$ joining $p_{2 j}$ and $q_{2 j}$, and $L_{j}$ bound a simply-connected domain $D_{j}$ on $\bar{R}-\partial \vec{R}$; we can assume that the domains $D_{1}, \cdots, D_{A}$ have pairwise disjoint closures on $\bar{R}$. Hence, there is a conformal mapping $f_{j}$ of $\mathrm{Cl} D_{j}$ onto $\left\{z=x+i y: 0 \leqslant x \leqslant a_{j}\right.$ and $\left.0 \leqslant y \leqslant b_{j}\right\}$ so that $f_{j}\left(p_{1 j}\right)=0, f_{j}\left(p_{2 j}\right)=i b_{j}, f_{j}\left(q_{1 j}\right)=a_{j}$ and $f_{j}\left(q_{2 j}\right)=$ $a_{j}+i b_{j}\left(0<a_{j}, b_{j}\right)$.

Considering a canonical exhaustion of $\bar{R}-\partial \bar{R}$ we conclude that for each $k=A+1, \cdots, B$ there is an analytic Jordan curve $L_{k}$ in $\bar{R}-\partial \bar{R}$ such that $L_{k}$ separates $\alpha_{1}(k)$ and $J_{k}$, and such that $J_{k}$ and $L_{k}$ bound a doubly-connected domain $D_{k}$ on $\bar{R}-\partial \vec{R}$. Let $f_{k}$ denote a conformal mapping of $\mathrm{Cl} D_{k}$ onto $\left\{z: 1<|z|<d_{k}\right\}$.

We can assume that $D_{1}, \cdots, D_{B}$ have pairwise disjoint closures on $\vec{R}$.

Let $G$ denote the family of all curves in $D=\bigcup_{j=1}^{B} D_{j}$ defined by $l \in G$ if for some $k, l \subset \mathrm{Cl} D_{k}-\gamma$ and $l$ joins $L_{k}$ to $J_{k}$. Since each curve in $F$ contains a curve in $G, \lambda(G) \leqslant \lambda(F)$. Also, $\lambda(G)>0$. Thus, $\lambda(F)>0$ and the sequence $\left\{D_{R_{n}}\left(u_{n}\right)\right\}$ converges to a finite limit.

Take $n<m$. Then 


$$
D_{R_{n}}\left(u_{m}, u_{n}\right)=\int_{\partial R_{n}} u_{m}^{*} d u_{n}=\int_{\alpha_{1 n}} * d u_{n}=D_{R_{n}}\left(u_{n}\right) \text {. }
$$

Hence,

$$
\begin{array}{r}
0<D_{R_{n}}\left(u_{m}-u_{n}\right)=D_{R_{n}}\left(u_{m}\right)-D_{R_{n}}\left(u_{n}\right)<D_{R_{m}}\left(u_{m}\right)-D_{R_{n}}\left(u_{n}\right) \\
\text { for } n<m .
\end{array}
$$

Therefore,

$$
D_{R_{n}}\left(u_{m}-u_{n}\right) \rightarrow 0 \text { as } m, n \rightarrow \infty(m \geqslant n) .
$$

Let $Q_{N}$ denote the double of $R_{N}$ at $\alpha_{0 N}-\mathrm{Cl} \gamma_{N}$. Extend each $u_{n}$, $n \geqslant N$, to a harmonic function $u_{n}^{\prime}$ on $Q_{N}[2$, p. 129]. From Theorem 1, with $z_{0} \in \alpha_{0 N}-\mathrm{Cl} \gamma_{N}$, there exists a harmonic function $v_{N}$ on $Q_{N}$ with the following properties:

(i) $D_{Q_{N}}\left(u_{n}^{\prime}-v_{N}\right) \rightarrow 0$ as $n \rightarrow \infty$,

(ii) $D_{Q_{N}}\left(u_{n}^{\prime}\right) \rightarrow D_{Q_{N}}\left(v_{N}\right)$ as $n \rightarrow \infty$,

(iii) $u_{n}^{\prime}(z) \rightarrow v_{N}(z)$ for $z \in Q_{N}$, and the convergence is uniform on every compact subset of $Q_{N}$.

It is easily seen after a similar reflection across $\gamma \cup \gamma^{*}\left(\gamma^{*}\right.$ is the reflection of $\boldsymbol{\gamma}$ in the first doubling process) that ${ }^{*} d v_{N}=0$ along $\gamma \cup \gamma^{*}$.

From (iii) it follows that $v_{N}$ is the restriction to $Q_{N}$ of a harmonic function $v$ on $Q$, the double of $\bar{R}$ across $\alpha_{0}-\mathrm{Cl} \gamma$.

We must show that

$$
\lim D_{Q_{N}}\left(u_{N}^{\prime}\right)=D_{Q}(v)=\lim D_{Q_{N}}\left(v_{N}\right) .
$$

This follows, since for $n \geqslant N, D_{Q_{n}}\left(u_{n}^{\prime}\right) \geqslant D_{Q_{N}}\left(u_{n}^{\prime}\right)$ and $D_{Q_{N}}\left(u_{N}^{\prime}\right)<D_{Q_{N}}\left(u_{n}^{\prime}\right)$

For the harmonic function $u=v \mid \bar{R}$,

(i) $u\left|R_{N}=v_{N}\right| R_{N}$,

(ii) $D_{R_{n}}\left(u_{n}-u\right) \rightarrow 0$ as $n \rightarrow \infty$,

(iii) $D_{R_{n}}\left(u_{n}\right) \rightarrow D_{\bar{R}}(u)$ as $n \rightarrow \infty$,

(iv) ${ }^{*} d u=0$ along $\gamma$,

(v) $u_{n}(z) \rightarrow u(z)$ for $n \rightarrow \infty$ and $z \in \bar{R}$, and the convergence is uniform on every compact subset of $\bar{R}$.

6. Second result. Theorem 6 will show that if $\alpha_{0}, \alpha_{1}$ and $\alpha_{2}$ are certain closed subsets of $\partial S$, then the conclusion of Theorem 5 is valid.

THEOREM 6. Let $S$ denote the compactification of $\bar{R}$ defined above. Set $\beta=S-\bar{R}$ and $\partial S=\beta \cup \partial \vec{R}$ Suppose that $\alpha_{0}, \alpha_{1}$ and $\alpha_{2}$ are closed subsets of $\partial S$ which satisfy the following conditions: 
(6.1) $\alpha_{0}$ and $\alpha_{1}$ each contain an arc on $\partial S$ or a type I contour of $\bar{R}$;

(6.2) $\alpha_{0}, \alpha_{1}$ and $\alpha_{2}$ are composed of points of $\beta$, type I contours of $\bar{R}$ and a finite number of arcs on $\partial S$;

(6.3) $\alpha_{0}, \alpha_{1}$ and $\alpha_{2}$ are painwise disjoint;

(6.4) For each $\beta^{\prime} \in\left(\alpha_{0} \cup \alpha_{1} \cup \alpha_{2}\right) \cap(\mathrm{Cl}(\partial \bar{R})-\partial \bar{R})$ there is a neighborhood of $\beta^{\prime}$ on $S$ which fails to meet $\beta-\left(\alpha_{0} \cup \alpha_{1} \cup \alpha_{2}\right)$.

Set $\gamma=\partial S-\left(\alpha_{0} \cup \alpha_{1} \cup \alpha_{2}\right)$ and denote by $F$ the family of all curves in $S-\gamma$ which join $\alpha_{0}$ to $\alpha_{1}$. Let $\beta^{*}=\left(\mathrm{Cl}(\partial \bar{R})-\left(\alpha_{0} \cup \alpha_{1} \cup \alpha_{2} \cup \partial \bar{R}\right)\right)$. There exists a continuous function $u$ on $S-\beta^{*}$ which is harmonic on $\bar{R}, 0$ on $\alpha_{0}, 1$ on $\alpha_{1}$, a constant on each component $\nu_{j}$ of $\alpha_{2}$ with the flux of. $u$ across $\nu_{j}$ equal to 0 and ${ }^{*} d u=0$ along $\gamma$ such that $0<\lambda(F)=D_{\bar{R}}(u)^{-1}<\infty$.

The conditions imposed upon $u \mid(S-\vec{R})$ are understood to be as limits in a sense to be made precise below.

PROOF. Without loss of generality assume that $\alpha_{0} \cup \alpha_{1} \cup \alpha_{2}$ contains a component $\delta^{\prime}$ with $\delta^{\prime}-\beta \neq 0$ and $\delta^{\prime} \cap \beta \neq 0$. Let $\left\{R_{n}\right\}$ denote a pseudosymmetric exhaustion of $\bar{R}$ and $\delta$ any component of $\alpha_{0} \cup \alpha_{1} \cup \alpha_{2}$ that intersects $\partial \bar{R}$. If $\delta \subset \partial \bar{R}$, assume that $\delta \subset \partial R_{1}$. If not, as we traverse $\partial \bar{R}$ in the positive sense we traverse the type II contours $\sigma_{1}, \cdots, \sigma_{q}$ of $\bar{R}$ which intersect $\delta$.

Consider the case where $\sigma_{j}-\delta \neq 0$ for $j=1$ or $q$. Without loss of generality assume that $\sigma_{q}-\delta \neq 0$ and let $f_{k}(k=1$ and $q)$ denote a homeomorphism of $\sigma_{k}$ onto $\{t: 0<t<1\}$. If $\sigma_{1}-\delta \neq 0$, there is a constant $a_{1}\left(0<a_{1}<1\right)$ such that $f_{1}^{-1}\left(\left\{t: a_{1} \leqslant t<1\right\}\right)=\delta \cap \sigma_{1}$; if not, set $a_{1}=1 / 2$. Let $a_{q}\left(0<a_{q}<1\right)$ denote the constant such that $f_{q}^{-1}\left(\left\{t: 0<t \leqslant a_{q}\right\}\right)=$ $\delta \cap \sigma_{q}$ and set $\epsilon=\min \left(a_{1}, 1-a_{1}, a_{q}, 1-a_{q}\right)$. Assume that $f_{k}^{-1}\left(\left\{t: a_{k}-\epsilon<\right.\right.$ $\left.\left.t<a_{k}+\epsilon\right\}\right) \subset \partial R_{1}$ for $k=1$ and $q$.

Set $\Gamma_{n}=\mathrm{Cl}\left(\partial R_{n}-\partial \bar{R}\right)$. Each component $\Gamma_{n}^{\prime}$ of $\Gamma_{n}-\partial \bar{R}$ is either an open analytic Jordan arc in $\bar{R}-\partial \bar{R}$ with end points on two distinct type II contours of $\bar{R}$ or a type I contour of $R_{n}$ in $\bar{R}-\partial \bar{R}$. If $\Gamma_{n}^{\prime}$ is a type I contour of $R_{n}$, let $C^{\prime}\left(\Gamma_{n}^{\prime}\right)$ denote the component of $S-\Gamma_{n}^{\prime}$ with $C^{\prime}\left(\Gamma_{n}^{\prime}\right) \cap \partial \bar{R}=0$. Without loss of generality assume that each $C^{\prime}\left(\Gamma_{n}^{\prime}\right)$ intersects at most one of the following sets: $\alpha_{0}, \alpha_{1}, \alpha_{2}$. If $C^{\prime}\left(\Gamma_{n}^{\prime}\right)$ intersects $\alpha_{k}(k=0,1$ or 2$)$ write $C\left(\Gamma_{n}^{\prime}, k\right)$ for $C^{\prime}\left(\Gamma_{n}^{\prime}\right)$, otherwise $C\left(\Gamma_{n}^{\prime}\right)$.

Suppose that $\Gamma_{n}^{\prime}$ is not a type I contour of $R_{n}$ and that our exhaustion satisfies the following remark, which is valid for some subsequence; let $A^{\prime}\left(\Gamma_{n}^{\prime}\right)$ denote the component of $S-\Gamma_{n}^{\prime}$ which satisfies either $A^{\prime}\left(\Gamma_{n}^{\prime}\right) \cap\left(\alpha_{0} \cup \alpha_{1} \cup \alpha_{2}\right)$ $\cap \mathrm{Cl}(\partial \bar{R})=0$ or for some $k=0,1$ or $2, A^{\prime}\left(\Gamma_{n}^{\prime}\right) \cap \alpha_{k} \cap \mathrm{Cl}(\partial \bar{R}) \neq 0$ and $A^{\prime}\left(\Gamma_{n}^{\prime}\right) \cap \alpha_{j} \cap \mathrm{Cl}(\partial \bar{R})=0$ for $j \neq k$. Write $A\left(\Gamma_{n}^{\prime}, k\right)$ for $A\left(\Gamma_{n}^{\prime}\right)$ if the latter occurs and $B\left(\Gamma_{n}^{\prime}\right)$ if the former. 
We can assume that $A\left(\Gamma_{n}^{\prime}, k\right) \cap\left(\bigcup_{j=0}^{2} \alpha_{j}-\alpha_{k}\right)=0$ and $B\left(\Gamma_{n}^{\prime}\right) \cap\left(\bigcup_{j=0}^{2} \alpha_{j}\right)=0$. Further, from (6.4), we can assume that each $A\left(\Gamma_{n}^{\prime}, k\right)$ fails to meet $\gamma-\partial \vec{R}$.

We have associated with each component $\Gamma_{n}^{\prime}$ of $\Gamma_{n}$ some $A\left(\Gamma_{n}^{\prime}, k\right), B\left(\Gamma_{n}^{\prime}\right)$, $C\left(\Gamma_{n}^{\prime}\right)$ or $C\left(\Gamma_{n}^{\prime}, k\right)$ domain on $S$. For each $k=0,1$ and 2 let $\Gamma_{n}(k)$ denote the union of all components $\Gamma_{n}^{\prime}$ of $\Gamma_{n}$ which are associated with some $A\left(\Gamma_{n}^{\prime}, k\right)$ domain and let $\Gamma_{C n}(k)$ denote the union of all components $\Gamma_{n}^{\prime}$ of $\Gamma_{n}$ which are associated with some $C\left(\Gamma_{n}^{\prime}, k\right)$ domain. Take $n \geqslant j \geqslant 1$. Set $\Gamma_{A j n}(k)=\left\{\Gamma^{\prime}: \Gamma^{\prime}\right.$ is a component of $\Gamma_{C n}(k)$ and $\Gamma^{\prime} \subset A\left(\Gamma_{j}^{\prime}, k\right)$ for some component $\Gamma_{j}^{\prime}$ of $\Gamma_{j}(k)$ \} and $\Gamma_{\gamma j}=\Gamma_{j}-\bigcup_{L=0}^{2} \Gamma_{j}(L)$. Let $M_{\gamma j}$ denote the totality of all $B\left(\Gamma_{j}^{\prime}\right), C\left(\Gamma_{j}^{\prime}\right)$, $C\left(\Gamma_{j}^{\prime}, 0\right), C\left(\Gamma_{j}^{\prime}, 1\right)$ and $C\left(\Gamma_{j}^{\prime}, 2\right)$ domains, where $\Gamma_{j}^{\prime}$ is a component of $\Gamma_{\gamma j}$.

For $k=0,1$ and 2 set

$S_{j n}=R_{n}-M_{\gamma j}, \alpha_{k n}(A)=\alpha_{k} \cap \partial S_{j n} \cap \partial \bar{R} \quad\left(=\alpha_{k} \cap \partial R_{n} \cap \partial \bar{R}\right), \alpha_{k j n}=$ $\alpha_{k n}(A) \cup \Gamma_{n}(k) \cup \Gamma_{C j}(k) \cup \Gamma_{A j n}(k)$, and

$\gamma_{j n}=\partial S_{j n}-U_{L=0}^{2} \alpha_{L j n}$.

Set $T_{j}=S-M_{\gamma j}, \alpha_{k j}=\left(\alpha_{k} \cap T_{j}\right) \cup \Gamma_{C j}(k)$ and

$\gamma_{j}=\left(T_{j} \cap \gamma\right) \cup\left(\Gamma_{\gamma j}-\bigcup_{L=0}^{2} \Gamma_{C j}(L)\right)$. In the sense of limits along curves tending to points of $\alpha_{k} \cap \beta \cap T_{j}$ and $\gamma \cap \beta \cap T_{j}, \alpha_{k j}=\lim _{n \rightarrow \infty} \alpha_{k j n}, \gamma_{j}=$ $\lim _{n \rightarrow \infty} \gamma_{j n}$ and $T_{j}=\lim _{n \rightarrow \infty} S_{j n}$.

For fixed $j$, let $F_{j n}$ denote the family of all curves in $S_{j n}-\gamma_{j n}$ which join $\alpha_{0 j n}$ to $\alpha_{1 j n}$ and let $u_{j n}$ denote the harmonic function on the compact bordered Riemann surface $S_{j n}$ satisfying Theorem 2 . Let $F_{j}$ denote the family of all curves in $T_{j}-\gamma_{j}$ which join $\alpha_{0 j}$ to $\alpha_{1 j}$. Clearly,

$$
\lambda\left(F_{j}\right) \geqslant \lambda\left(F_{j n+1}\right) \geqslant \lambda\left(F_{j n}\right) \geqslant \lambda\left(F_{j 1}\right)>0 .
$$

Also, since the family $G_{j}$ of all curves in $T_{j}-\left(\beta \cup \gamma_{j}\right)$ which join $\alpha_{0 j}-\beta$ to $\alpha_{1 j}-\beta$ is a subset of $F_{j}$, from Theorem $5, \lambda\left(F_{j}\right) \leqslant \lambda\left(G_{j}\right)<\infty$. Hence,

$$
0<\lim _{n \rightarrow \infty} \lambda\left(F_{j n}\right) \leqslant \lambda\left(F_{j}\right)<\infty .
$$

To establish the inequality $\lim _{n \rightarrow \infty} \lambda\left(F_{j n}\right) \geqslant \lambda\left(F_{j}\right)$ we follow the procedure of Marden and Rodin. [5, pp. 151-156] by defining a family $F_{j 0}$ in $T_{j}$ such that $\lambda\left(F_{j 0}\right)=\lambda\left(F_{j}\right)$ and $\lambda\left(F_{j 0}\right)=\lim _{n \rightarrow \infty} \lambda\left(F_{j n}\right)$.

Take $l_{N} \in F_{j N}$ and $n \leqslant N$ so that $l_{N}$ intersects

$$
\bigcup_{L=0}^{2}\left(\left(\alpha_{L N}(A)-\alpha_{L n}(A)\right) \cup \Gamma_{N}(L) \cup \Gamma_{A j N}(L)\right) \text {. }
$$

We will define a type of restriction of $l_{N}$ to $S_{j n}$, denoted by $l_{N} \| S_{j n}$, with the property that $l_{N} \| S_{j n} \in F_{j n}$. Assume that $l_{N}$ is parametrized so that $l_{N}(0) \in$ $\alpha_{0 j N}$ and $l_{N}(1) \in \alpha_{1 j N}$. There is a greatest $t$ such that $l_{N}(t) \in \alpha_{0 j n}$; call it $t_{1}$. Suppose that $l_{N}$ intersects $\alpha_{2 j n}-\alpha_{2 n}(A)$. Let $t_{2}$ be the smallest $t$ such 
that $l_{N}(t) \in \alpha_{2 j n}-\alpha_{2 n}(A)$ and denote by $C_{2}$ the component of $\alpha_{2 j n}-$ $\alpha_{2 n}(A)$ which contains $l_{N}\left(t_{2}\right)$. Let $t_{3}$ be the greatest $t$ such that $l_{N}(t) \in C_{2}$. Continue in this manner defining points $t_{2 k}, t_{2 k+1}$ such that $t_{2 k}$ is the smallest $t$ and $t_{2 k+1}$ the largest $t$ such that $l_{N}(t)$ lies on a component $C_{2 k}$ of $\alpha_{2 j n}-\alpha_{2 n}(A)$ until we define $t_{m-2}, t_{m-1}(m \geqslant 4$ and even) such that $l_{N} \mid\left\{t: t_{m-1}<t \leqslant 1\right\} \cap\left(\alpha_{2 j n}-\alpha_{2 j N}\right)=0$. Letting $t_{m}$ be the smallest $t$ such that $l_{N}(t) \in \alpha_{1 j n}$ and $C_{(m+2) / 2}$ the component of $\alpha_{1 j n}$ which contains $l_{N}\left(t_{m}\right)$, we obtain an even number of stopping times $0 \leqslant t_{1}<\cdots<t_{m} \leqslant 1$, a sequence of stopping points $l_{N}\left(t_{1}\right), \cdots, l_{N}\left(t_{m}\right)$ and a component sequence $C_{1}, \cdots, C_{(m+2) / 2}$ of distinct components of $\alpha_{0 j n} \cup \alpha_{1 j n} \cup\left(\alpha_{2 j n}-\alpha_{2 n}(A)\right)$.

Suppose that $l_{N}$ does not intersect $\alpha_{2 j n}-\alpha_{2 n}(A)$. Let $t_{2}$ be the smallest $t$ such that $l_{N}(t) \in \alpha_{1 j n}$ and let $C_{2}$ denote the component of $\alpha_{1 j n}$ which contains $l_{N}\left(t_{2}\right)$. We obtain the stopping times $0 \leqslant t_{1}<t_{2} \leqslant 1$, stopping points $l_{N}\left(t_{1}\right), l_{N}\left(t_{2}\right)$ and a component sequence $C_{1}, C_{2}$. Necessarily, by our assumption on $l_{N}, t_{1}=0$ and $t_{2}=1$ is impossible.

In each case define $l_{N} \| S_{j n}$ to be the restriction of $l_{N}$ to $\left[t_{1}, t_{2}\right] \cup$ $\left[t_{3}, t_{4}\right] \cup \cdots \cup\left[t_{m-1}, t_{m}\right]$.

A 1-chain $l_{0}$ on $T_{j}$ will belong to $F_{j 0}$ if either $l_{0}=l$ for some $l \in F_{j}$ or if $l_{0}$ is a continuous map of an open dense subset of $[0,1]$ into $T_{j}$ such that:

(i) If $t_{0}$ is not in the domain dom $l_{0}$ of $l_{0}$ and $0<t_{0}<1$, then there exist sequences $\left\{r_{k}\right\},\left\{s_{k}\right\}$ in dom $l_{0}$ such that $r_{k} \uparrow t_{0}, s_{k} \downarrow t_{0}$, and a point $\beta_{0} \in \beta \cap T_{j}$ with $l_{0}\left(r_{k}\right) \rightarrow \beta_{0}, l_{0}\left(s_{k}\right) \rightarrow \beta_{0}$. If $t_{0}=0$ (respectively 1) we require only a sequence $\left\{s_{k}\right\}$ (respectively $\left\{r_{k}\right\}$ ).

(ii) There exists a subsequence $\left\{S_{j n(k)}\right\}$ of $\left\{S_{j n}\right\}$ such that $l_{0} \| S_{j n(k)} \equiv$ $\left(l_{0} \mid S_{j n(k)}\right) \| S_{j n(k)} \in F_{j n(k)}$ for all $k \geqslant 1$.

(iii) If $t \in \operatorname{dom} l_{0}$ then there exists a $p$ such that $t \in \operatorname{dom} l_{0} \| S_{j n(k)}$ for all $k \geqslant p$.

We will now show that $\lambda\left(F_{j 0}\right)=\lambda\left(F_{j}\right)$. If a 1-chain $l_{0} \in F_{j 0}$ can be extended to $\{t: 0<t<1\}$ with values in $T_{j}$, then the extension will be in $F_{j}$. Let $\left\{\xi_{n}(p)\right\}_{p=1}^{V_{n}}$ denote the components of $\bigcup_{L=0}^{2} \Gamma_{n}(L)$. To each $\xi_{n}(p)$ we associate a simply-connected domain $D_{n p}$ in $S_{j n}$ bounded by $\xi_{n}(p)$ and an open analytic Jordan arc $\tau_{n p}$ in $S_{j n}-\partial S_{j n}$ with end points on $\partial \vec{R} \cap S_{j n}$, and a homeomorphism $f_{n p}$ of $\mathrm{Cl} D_{n p}$ onto $\left\{z=x+i y: 0 \leqslant x \leqslant a_{n p}, 0 \leqslant\right.$ $\left.y \leqslant b_{n p}\right\}, 0<a_{n p}, b_{n p}$, which is conformal on $D_{n p}-\partial \bar{R}$ with $f_{n p}\left(\xi_{n}(p)\right)=$ $\left\{x: 0 \leqslant x \leqslant a_{n p}\right\}, f_{n p}\left(\tau_{n p}\right)=\left\{x+i b_{n p}: 0<x<a_{n p}\right\}$ and $f_{n p}\left(\partial R \cap D_{n p}\right)=$ $\left\{i y: 0<y<b_{n p}\right\} \cup\left\{a_{n p}+i y: 0<y<b_{n p}\right\}$.

Let $\left\{\xi_{n}(p)\right\}_{p}^{W_{n}} V_{n}+1$ denote the components of $\bigcup_{L=0}^{2} \Gamma_{A j n}(L)$. To each $\xi_{n}(p), p=V_{n}+1, \cdots, W_{n}$, we associate an annular domain $D_{n p}$ in $S_{j n}$ bounded by $\xi_{n}(p)$ and an analytic Jordan curve $\tau_{n p}$ in $S_{j n}-\partial S_{j n}$, and a 
homeomorphism $f_{n p}$ of $\mathrm{Cl} D_{n p}$ onto $\left\{z: a_{n p} \leqslant|z| \leqslant b_{n p}\right\}, 0<a_{n p}<b_{n p}$, which is conformal on $D_{n p}$ with $f_{n p}\left(\xi_{n}(p)\right)=\left\{z:|z|=a_{n p}\right\}$ and $f_{n p}\left(\tau_{n p}\right)=$ $\left\{z:|z|=b_{n p}\right\}$. We can assume that the domains $D_{n p}, p=1, \cdots, W_{n}$, have pairwise disjoint closures on $S_{j n}$.

We shall show that if no $D_{n p}$ is crossed infinitely many times by $l_{0}$ then $l_{0}$ can be extended continuously to $\{t: 0<t<1\}$. Choose $t_{0} \notin$ dom $l_{0}$ with $0<t_{0}<1$. Let $\left\{r_{m}\right\},\left\{s_{m}\right\}$ and $\beta_{1} \in \beta \cap T_{j}$ be as above. It suffices to show that $l_{0}(t) \rightarrow \beta_{1}$ for $t \rightarrow t_{0}$ and $t \in \operatorname{dom} l_{0}$.

Let $G$ denote a neighborhood of $\beta_{1}$ on $T_{j}$. We want a neighborhood of $t_{0}$ whose $l_{0}$-image is in a "small" neighborhood of $G$. To do this we assume that $G$ is a component of $T_{j}-S_{j N}$ for some $N$. Let $D_{N p}$ be one of the domains described above for $\xi_{N}(p)=\mathrm{Cl} G-G$. For $n$ sufficiently large, $l_{0}\left(r_{n}\right), l_{0}\left(s_{n}\right) \in G$.

If $l_{0}(t) \nrightarrow \beta_{1}$ as $t \rightarrow t\left(t \in \operatorname{dom} l_{0}\right)$ for some domains $G$ and $D_{N p}$, there would exist a sequence $\left\{v_{k}\right\}$ in $\operatorname{dom} l_{0}$ with $v_{k} \rightarrow t_{0}$ and $l_{0}\left(v_{q}\right) \notin$ $G \cup D_{N p}$ for each $q \geqslant 1$. A subsequence of $\left\{v_{k}\right\}$ is monotone, say $v_{k} \uparrow t_{0}$. Choose $r_{k(1)}$ and $v_{k(1)}>r_{k(1)}$ and $S_{j m(1)} \supset S_{j N}$ so that $r_{k(1)}, v_{k(1)} \in$ $\operatorname{dom} l_{0} \| S_{j m(1)}$. Since $l_{0} \| S_{j m(1)} \in F_{j m(1)}$, there is a crossing of $D_{N p}$ within $\left\{t: r_{k(1)}<t<v_{k(1)}\right\}$. Next choose $r_{k(2)}, v_{k(2)}, S_{j m(2)}$ so that $r_{k(1)}<v_{k(1)}$ $<r_{k(2)}<v_{k(2)}$ and $r_{k(2)}, v_{k(2)} \in \operatorname{dom} l_{0} \| S_{j m(2)}$. There must be a crossing of $D_{N p}$ within $\left\{t: r_{k(2)}<t<v_{k(2)}\right\}$. In this way we see that $l_{0}$ crosses $D_{N p}$ infinitely many times. The cases $t_{0}=0$ and $t_{0}=1$, if they arise, are handled analogously.

Let $F_{j 0}(n)$ denote the family of all curves in $F_{j 0}$ which cross some $D_{n p}$ infinitely many times. Clearly, $\lambda\left(F_{j 0}(n)\right)=\infty$.

Let $F_{j 0}^{\prime}$ denote the family of all curves in $F_{j 0}$ which cannot be continuously extended to $\{t: 0<t<1\}$. Clearly, $F_{j 0}^{\prime} \subset \bigcup_{n \geqslant j} F_{j 0}(n)$. So $\lambda\left(F_{j 0}^{\prime}\right)=\infty$. $\lambda\left(F_{j}\right)$.

Now, $F_{j 0}=F_{j 0}^{\prime} \cup\left(F_{j 0}-F_{j 0}^{\prime}\right)$. So $\lambda\left(F_{j 0}\right)=\lambda\left(F_{j 0}-F_{j 0}^{\prime}\right)$ and $\lambda\left(F_{j 0}\right)=$

We are now ready to show that $\lim _{n \rightarrow \infty} \lambda\left(F_{j n}\right) \geqslant \lambda\left(F_{j 0}\right)$. Choose any $x<\lambda\left(F_{j 0}\right)$ and any metric $\rho|d z|$ on $T_{j}$ such that $L^{2}\left(F_{j 0}, \rho|d z|\right)>x$ and $A\left(\rho|d z|, T_{j}\right)=1$. To show that $\lim _{n \rightarrow \infty} \lambda\left(F_{j n}\right) \geqslant \lambda\left(F_{j 0}\right)$ it suffices to show that

$$
\lim _{n \rightarrow \infty} L^{2}\left(F_{j n}, \rho|d z|\right) \geqslant x \text {. }
$$

If that failed to hold there would exist a subsequence along which $L^{2}\left(F_{j n}, \rho|d z|\right)$ had a limit $y<x$. If we can show that to each $\epsilon>0$ there exists a $l(\epsilon) \in F_{j 0}$ satisfying $\left(\int_{l(\epsilon)} \rho|d z|\right)^{2} \leqslant y+7 \epsilon$, then we have the desired contradiction,

$$
y<x<L^{2}\left(F_{j 0}, \rho|d z|\right) \leqslant\left(\int_{l(\epsilon)} \rho|d z|\right)^{2} \leqslant y+7 \epsilon .
$$


Fix $\epsilon>0$. By passage to a subsequence of $\left\{F_{j n}\right\}$ we may assume that

$$
\left|L^{2}\left(F_{j n}, \rho|d z|\right)-y\right|<\epsilon / 2^{n} \quad(n \geqslant 1) .
$$

Whenever a subsequence of $\left\{F_{j n}\right\}$ or $\left\{l_{n}: l_{n} \in F_{j n}\right\}$ is extracted and the notation is unchanged we tacitly agree that $\left\{S_{j n}\right\}$ shall refer to the corresponding subsequence of $\{n\}$.

Choose $l_{n} \in F_{j n}$ so that

$$
\left|\left(\int_{l_{n}} \rho|d z|\right)^{2}-y\right|<\epsilon / 2^{n} \quad(n \geqslant 1) .
$$

From the sequence $\left\{l_{n}\right\}$, possibly after some modifications, we will extract a subsequence which will be used to construct $l(\epsilon)$.

If there is an $M$ such that

$$
\left\{l_{M}(t) \cap \partial S_{j M}: 0 \leqslant t \leqslant 1\right\} \subset\left(\alpha_{0} \cup \alpha_{1} \cup \alpha_{2}\right) \cap \partial \bar{R},
$$

take $l(\epsilon)=l_{M} \in F_{j 0}$; the assertion follows. Therefore, assume that this is not the case. We first find a subsequence $\left\{l_{n(k)}\right\}$ of $\left\{l_{n}\right\}$ such that all $l_{n(k)} \| S_{j N}$, $n(k) \geqslant N$, have the same component sequence on $\partial S_{j N}$. Since there are only a finite number of possible component sequences on $\partial S_{j j}$ we may select a first subsequence of $\left\{l_{n}\right\}$, all elements of which have the same component sequence on $\partial S_{j j}$. By induction we obtain for each $N \geqslant j$ a subsequence of the preceding one, all elements of which follow a common component sequence on $\partial S_{j N}$. Using the diagonal process we obtain a subsequence with the desired property. Continue to designate this subsequence by $\left\{l_{n}\right\}$.

Now modify each $l_{n}$ so that not only will all $l_{n} \| S_{j N}, n \geqslant N$, follow the same component sequence, but also $l_{n} \| S_{j n-1}$ and $l_{n-1} \| S_{j n-1}$ will have the same sequence of stopping points on $\partial S_{j n-1}$. To accomplish this use the diagonal process to find a preliminary sequence, again denoted by $\left\{l_{n}\right\}$, with the following property. Suppose that $l_{N}$ has $m$ stopping points on $\partial S_{j N}$. Then for each $k \leqslant m$ the $k$ th stopping point $\zeta_{n}$ of $l_{n} \| S_{j N}, n \geqslant N$, gives rise to a convergent sequence of points $\left\{\zeta_{n}\right\}$ on a component of $\bigcup_{L=0}^{2}\left(\alpha_{L j N}-\alpha_{L N}(A)\right)$. Around the limit point of this sequence put a topological disc the circumference of which has very small $\rho$-length.

For each $N$ there are as many discs on $\partial S_{j N}$ as there are stopping points for $l_{n} \| S_{j N}, n \geqslant N$. Choose the circumference of these discs small enough so that their total $\rho$-length is less than $2^{-N} \epsilon$. By the diagonal process we achieve a situation in which each stopping point of $l_{n} \| S_{j N}$ on $\partial S_{j n}$ is inside the appropriate disc for all $n \geqslant N$. For each disc take a point in the intersection of its circumference and the corresponding component of the component sequence; call such 
a point a distinguished stopping point. A modification of $l_{n}$ will mean the result of replacing part of its path inside a disc by a path on the circumference of the disc. Modify $l_{N}$ so that all its stopping points are distinguished; in general, modify $l_{n}$ so that the stopping points of $l_{n} \| S_{j n-1}$ on $\partial S_{j n-1}$ and $l_{n} \| S_{j n}$ on $\partial S_{j n}$ are distinguished. Denote the modified sequence again by $\left\{l_{n}\right\}$. This modification has increased the $\rho$-length of $l_{n}$ by at most $2^{-n+1} \epsilon+2^{-n} \epsilon$. For the present sequence $\left\{l_{n}\right\}$,

$$
\left|\left(\int_{l_{M}} \rho|d z|\right)^{2}-y\right|<\frac{\epsilon}{2^{n}}+\frac{\epsilon}{2^{n-1}}+\frac{\epsilon}{2^{n}}=\frac{4 \epsilon}{2^{n}} .
$$

Clearly, each new $l_{n}$ is in $F_{j n}$.

By induction, for each $n$ we reparametrize $l_{n}$ so that $\operatorname{dom} l_{n} \| S_{j n-1}=$ dom $l_{n-1}$. So dom $l_{n-1}$ consists of a finite number of closed intervals $\left[t_{1}, t_{2}\right]$ $\cup\left[t_{3}, t_{4}\right] \cup \cdots \cup\left[t_{m-1}, t_{m}\right]$ and $l_{n-1}\left(t_{k}\right)=l_{n}\left(t_{k}\right)$ for $1 \leqslant k \leqslant m$.

We now construct $l(\epsilon)$. On $\operatorname{dom} l_{1}$ set $l(\epsilon)=l_{1}$. In general, if $l(\epsilon)$ has been defined on $\operatorname{dom} l_{n-1}$ set $l(\epsilon)=l_{n}$ on $\operatorname{dom} l_{n}-\operatorname{dom} l_{n-1}$. So $l(\epsilon)$ is a curve on $T_{j}$. Its domain is an open subset of $[0,1]$ which, by reparametrization, may be assumed to be dense.

Note, since $l_{n} \mid$ dom $l_{n-1}=l_{n} \| S_{j n-1}$,

$$
\left(\int_{l_{n} \| S_{j n-1}} \rho|d z|\right)^{2} \geqslant L^{2}\left(F_{j n-1}, \rho|d z|\right)>y-\epsilon / 2^{n-1} .
$$

Also, $\left(\int_{l_{n}} \rho|d z|\right)^{2}<y+4 \epsilon / 2^{n}$. Hence,

$$
\left(\int_{l_{n} \mid\left(\operatorname{dom} l_{n}-\operatorname{dom} l_{n-1}\right)} \rho|d z|\right)^{2}<\frac{6 \epsilon}{2^{n}}
$$

Therefore, by Schwarz's inequality,

$$
\left(\int_{l(\epsilon)} \rho|d z|\right)^{2}<\left(\int_{l_{1}} \rho|d z|\right)^{2}+\sum_{n=1}^{\infty} \frac{6 \epsilon}{2^{n}}<y+7 \epsilon .
$$

Clearly, see $\left[5\right.$, p. 225], $l(\epsilon) \in F_{j 0}$. Hence,

$$
0<\lambda\left(F_{j}\right)=\lim _{n \rightarrow \infty} D_{s_{j n}}\left(u_{j n}\right)^{-1}<\infty .
$$

Take $m \leqslant n$. We will show that $D_{s_{j m}}\left(u_{j m}-u_{j n}\right) \rightarrow 0$ as $m, n \rightarrow \infty$. Note, since $\gamma_{j m} \subset \gamma_{j n}, u_{j m}$ is a constant on each component $\Gamma^{\prime}$ of $\Gamma_{C j}(2)$ and the flux of $u_{j m}$ along $\Gamma^{\prime}$ is 0 ,

$$
\begin{aligned}
D_{S_{j m}}\left(u_{j m}, u_{j n}\right) & =\int_{\alpha_{1 j m}} * d u_{j n}+\int_{\alpha_{2 j m}} u_{j m} * d u_{j n} \\
& =\int_{\alpha_{1 j m}} * d u_{j n}+\int_{\alpha_{2 m}}(A)+\Gamma_{m}(2)+\Gamma_{A j m}(2) u_{j m} * d u_{j n} .
\end{aligned}
$$


Set $k=1$ and 2. For each component $\delta$ of $\Gamma_{p}(k)$ let $A(\delta, k)$ denote the domain on $S$ associated with $\delta$. Define $\Lambda_{k p}(1)$ to be the union of all components $\delta$ of $\Gamma_{p}(k)$ for which $A(\delta, k) \cap \gamma \cap \partial \bar{R}=0, \Lambda_{k p}(2)$ to be the union of all components $\delta$ of $\Gamma_{p}(k)$ for which $(A(\delta, k) \cap \partial S)-(\gamma \cup \beta)$ is an open arc on $\partial \bar{R}$ and set $\Lambda_{k p}(3)=\Gamma_{p}(k)-\left(\Lambda_{k p}(1) \cup \Lambda_{k p}(2)\right)$.

For simplicity we shall write $\Gamma^{\prime} \in \Lambda_{k p}(m)$ or $\Gamma^{\prime} \in \Gamma_{A j p}(k)$ whenever we mean that $\Gamma^{\prime}$ is a component of that set.

For each $\delta$ in $\Lambda_{k p}(1)$ let $\alpha_{k p}(1, \delta)$ and $\alpha_{k p}(2, \delta)$ denote the two components of $\alpha_{k p}(A)$ for which $\alpha_{k p}(1, \delta) \cup \delta \cup \alpha_{k p}(2, \delta)$ is connected. For each $\delta$ in $\Lambda_{k p}(2)$ let $\alpha_{k p}(\delta)$ denote the component of $\alpha_{k p}(A)$ and $\gamma_{j p}(\delta)$ the component of $\gamma_{j p}$ for which $\alpha_{k p}(\delta) \cup \delta \cup \gamma_{j p}(\delta)$ is connected. Finally, for each $\delta$ in $\Lambda_{k p}(3)$ let $\gamma_{j p}(1, \delta)$ and $\gamma_{j p}(2, \delta)$ denote the components of $\gamma_{j p}$ for which $\gamma_{j p}(1, \delta) \cup \delta \cup \gamma_{j p}(2, \delta)$ is connected.

Let $E_{q}, q=1, \cdots, P$, denote the components of $S_{j n}-\left(S_{j m}-\partial S_{j m}\right)$. Set $\tau_{q}=\left\{\Gamma_{n}^{\prime} \in \Gamma_{A j n}(k): \Gamma_{n}^{\prime} \subset E_{q}\right\}, \Gamma_{A j m n}(k)=\bigcup_{q=1}^{P} \tau_{q}$ and for each $\Gamma^{\prime} \in \Gamma_{A j m}(k)$, let $\Gamma_{\sigma}\left(\Gamma^{\prime}\right), \sigma=1, \cdots, \omega$, denote the finite number of components of $\Gamma_{A j n}(k)-\Gamma_{A j m n}(k)$ such that $\left\{\Gamma^{\prime}, \Gamma_{1}\left(\Gamma^{\prime}\right), \cdots, \Gamma_{\omega}\left(\Gamma^{\prime}\right)\right\}$ bound a compact bordered Riemann surface $E$ on $S_{j n}$. left. Then

Orient each subarc of $\partial S_{j p}, p \geqslant 1$, so that $S_{j p}-\partial S_{j p}$ lies locally to the

$$
\int_{\Gamma^{\prime}} * d u_{j n}=\sum_{\sigma=1}^{\omega} \int_{\Gamma_{\sigma}\left(\Gamma^{\prime}\right)} * d u_{j n} \text { for } \Gamma^{\prime} \in \Gamma_{A j m}(k), m \leqslant n .
$$

Thus,

$$
\int_{\Gamma_{A j m}(1)}^{* d u_{j n}}=\int_{\Gamma_{A j n}(1)-\Gamma_{A j m n}(1)} * d u_{j n}
$$

and

$$
\int_{\Gamma_{A j m}(2)} * d u_{j n}=0
$$

We now consider the following three cases:

A. Suppose that $\delta_{m}(q) \in \Lambda_{k m}(1) \cap \partial E_{q}$. Then $E_{q}$ has positively oriented border

$$
\sum_{L=1}^{2}\left(\alpha_{k n}\left(L, \delta_{n}(q)\right)-\alpha_{k m}\left(L, \delta_{m}(q)\right)\right)+\delta_{n}(q)-\delta_{m}(q)+\tau_{q},
$$

where $\delta_{n}(q) \in \Lambda_{k n}(1) \cap \partial E_{q}$. Thus,

(4) $\int_{\delta_{m}(q)}^{* d u_{j n}}=\sum_{L=1}^{2} \int_{\alpha_{k n}}\left(L, \delta_{n}(q)\right)-\alpha_{k m}\left(L, \delta_{m}(q)\right){ }^{*} d u_{j n}+\int_{\delta_{n}(q)+\tau_{q}}^{* d u_{j n}}$. 
B. If $\delta_{m}(q) \in \Lambda_{k m}(2) \cap \partial E_{q}$, then

$\partial E_{q}=\alpha_{k n}\left(\delta_{n}(q)\right)-\alpha_{k m}\left(\delta_{m}(q)\right)+\delta_{n q}+\gamma_{j n}\left(\delta_{n}(q)\right)-\gamma_{j m}\left(\delta_{m}(q)\right)-\delta_{m}(q)+\tau_{q}$, where $\delta_{n}(q) \in \Lambda_{k n}(2) \cap \partial E_{q}$. Thus,

(5) $\quad \int_{\delta_{m}(q)} * d u_{j n}=\int_{\alpha_{k n}\left(\delta_{n}(q)\right)-\alpha_{k m}\left(\delta_{m}(q)\right)}{ }^{*} d u_{j n}+\int_{\delta_{n}(q)+\tau_{q}} * d u_{j n}$.

C. If $\delta_{m}(q) \in \Lambda_{k m}(3) \cap \partial E_{q}$, then

$$
\partial E_{q}=\sum_{L=1}^{2}\left(\gamma_{j n}\left(L, \delta_{n}(q)\right)-\gamma_{j m}\left(L, \delta_{m}(q)\right)\right)+\delta_{n}(q)-\delta_{m}(q)+\tau_{q},
$$

where $\delta_{n}(q) \in \Lambda_{k n}(3) \cap \partial E_{q}$. Thus,

$$
\int_{\delta_{m}(q)}{ }^{*} d u_{j n}=\int_{\delta_{n}(q)+\tau_{q}}{ }^{*} d u_{j n}
$$

Take $k=1$. From (4), (5) and (6) we conclude that

$$
\int_{\Gamma_{m}(1)}{ }^{*} d u_{j n}=\int_{\alpha_{1 n} \cdot(A)-\alpha_{1 m}(A)}{ }^{*} d u_{j n}+\int_{\Gamma_{n}(1)+\Gamma_{A j m n}(1)}{ }^{*} d u_{j n} .
$$

Thus, using (2), $\Gamma_{A j m n}(1) \subset \Gamma_{A j n}(1)$ and $\alpha_{1 m}(A) \subset \alpha_{1 n}(A)$,

$$
\int_{\alpha_{1 j m}} * d u_{j n}=\int_{\alpha_{1 j n}} * d u_{j n}=D_{S_{j n}}\left(u_{j n}\right) \text {. }
$$

We wish to show that

$$
\int_{\alpha_{2 m}(A)+\Gamma_{m}(2)+\Gamma_{A j m}(2)} u_{j m} * d u_{j n}=0 .
$$

Let $\alpha_{2}^{*}$ denote the noncompact components of $\alpha_{2}$ on $\mathrm{Cl}(\partial \bar{R})$ and set $\alpha_{2 m}(A)^{*}=\alpha_{2}^{*} \cap \alpha_{2 m}(A)$. Clearly, $\int_{\alpha_{2 m}(A)-\alpha_{2 m}(A) * u_{j m}}{ }^{*} d u_{j n}=0$. Also, since $u_{j m}$ is a constant on each component of $\Gamma_{A j m}(2)$, from (3), $\int_{\Gamma_{A j m}(2)} u_{j m}{ }^{*} d u_{j n}=0$. Hence, we need only show that

$$
\int_{\alpha_{2 m}(A) *+\Gamma_{m}(2)} u_{j m} * d u_{j n}=0 .
$$

For each $q=1, \cdots, P, \partial E_{q}$ can be decomposed as in A, B or C Fix any $q$. We consider the three decompositions of $\partial E_{q}$.

A. Suppose that $\alpha_{2 n}\left(1, \delta_{n}(q)\right)$ and $\alpha_{2 n}\left(2, \delta_{n}(q)\right)$ fail to meet any $A\left(2, \delta_{m}(r)\right), r \neq q$. Then $\alpha_{2 m}\left(1, \delta_{m}(q)\right), \alpha_{2 m}\left(2, \delta_{m}(q)\right) \in \alpha_{2 m}(A)^{*}$, $\alpha_{2}\left(1, \delta_{n}(q)\right) \cup \Gamma_{n}(q) \cup \alpha_{2 n}\left(2, \delta_{n}(q)\right)$ is a component of $\alpha_{2 j n}$ and $u_{j m}$ is a constant on 


$$
\xi_{m}=\alpha_{2 m}\left(1, \delta_{m}(q)\right) \cup \delta_{m}(q) \cup \alpha_{2 m}\left(2, \delta_{m}(q)\right) .
$$

Hence, $\int_{\xi_{m}} u_{j m} * d u_{j n}=0$.

Suppose that there is an $r \neq q$ so that $\alpha_{2 n}\left(1, \delta_{n}(q)\right) \cap A\left(2, \delta_{m}(r)\right) \neq 0$. Necessarily, $\partial E_{r} \cap \partial \bar{R}$ is composed of sets of the form $\alpha_{2 n}\left(1, \delta_{n}(r)\right)$ and $\alpha_{2 n}\left(2, \delta_{n}(r)\right)$ or $\alpha_{2 n}\left(\delta_{n}(r)\right)$ and $\gamma_{j n}\left(\delta_{n}(r)\right)$. For simplicity assume that the former occurs, that $\alpha_{2 n}\left(1, \delta_{n}(q)\right)=\alpha_{2 n}\left(1, \delta_{n}(r)\right)$ and that $\alpha_{2 n}\left(2, \delta_{n}(r)\right) \cap$ $A\left(2, \delta_{m}(t)\right)=0$ for $t \neq r$. As above, we conclude that $\int_{\xi_{m}} u_{j m}{ }^{*} d u_{j n}=0$, where

$$
\xi_{m}=\alpha_{2 m}\left(1, \delta_{m}(q)\right) \cup \delta_{m}(q) \cup \alpha_{2 m}\left(2, \delta_{m}(q)\right) \cup \delta_{m}(r) \cup \alpha_{2 m}\left(2, \delta_{m}(r)\right) .
$$

Cases B and C are handled analogously.

We have shown that $D_{s_{j m}}\left(u_{j n}, u_{j n}\right)=D_{s_{j n}}\left(u_{j n}\right)$ for $n \geqslant m$. Thus, $D_{s_{j m}}\left(u_{j m}-u_{j n}\right)<D_{s_{j m}}\left(u_{j m}\right)-D_{s_{j n}}\left(u_{j n}\right)$. Hence, since the sequence $\left\{D_{s_{j p}}\left(u_{j p}\right)\right\}$ has a finite limit, $D_{s_{j n}}\left(u_{j m}-u_{j n}\right) \rightarrow 0$ as $m, n \rightarrow \infty$.

To show that the functions $u_{j n}$ converge to a limit function $u_{j}$ on $T_{j}$ we proceed as in the proof of Theorem 5 by considering the double $Q_{N}$ of $S_{j N}$ at $\left(\alpha_{0 N}(A)-\mathrm{Cl}\left(\gamma_{j N}\right)\right) \cup \Gamma_{C j}(0)$ and the extensions $u_{j n}^{\prime}$ of $u_{j n}(n \geqslant N)$ on $Q_{N}$. There exists a harmonic function $v_{j N}^{\prime}$ on $Q_{N}$ with the following properties:

$$
\begin{aligned}
& D_{Q_{N}}\left(u_{j n}^{\prime}-v_{j N}^{\prime}\right) \rightarrow 0 \text { as } n \rightarrow \infty, \\
& D_{Q_{N}}\left(u_{j n}^{\prime}\right) \rightarrow D_{Q_{N}}\left(v_{j N}^{\prime}\right) \text { as } n \rightarrow \infty, \\
& u_{j n}^{\prime}(z) \rightarrow v_{j N}^{\prime}(z) \text { as } n \rightarrow \infty \text { for } z \in Q_{N},
\end{aligned}
$$

and the convergence is uniform on every compact subset of $Q_{N}$.

Let $D(\bar{R})$ denote the double of $T_{j}$ across $\left(\alpha_{0} \cup \Gamma_{C j}(0)\right)-(\beta \cup \mathrm{Cl} \gamma)$. Then $v_{j N}^{\prime}$ is the restriction to $Q_{N}$ of a continuous function $v_{j}$ on $D(\bar{R}) \cup$ $\left(T_{j} \cap\left(\beta-\beta^{*}\right)\right)$ which is harmonic on $D(\bar{R})$.

To show that $\lim D_{Q_{N}}\left(u_{j N}^{\prime}\right)=\lim D_{Q_{N}}\left(v_{j N}^{\prime}\right)$ it suffices to show that $\lim D_{S_{j N}}\left(u_{j N}\right)=\lim D_{S_{j N}}\left(v_{j N}\right), v_{j N}=v_{j N}^{\prime} \mid S_{j N}$.

Note that for $n \geqslant N$,

$$
D_{s_{j N}}\left(u_{j N}-u_{j n}\right)=D_{s_{j N}}\left(u_{j N}\right)-2 D_{s_{j n}}\left(u_{j n}\right)+D_{s_{j N}}\left(u_{j n}\right)
$$

tends to 0 as $n$ and $N$ tend to $\infty$. Thus,

$$
\lim _{n \rightarrow \infty} D_{s_{j N}}\left(u_{j N}-u_{j n}\right)=D_{s_{j N}}\left(u_{j N}\right)-2 \lim _{n \rightarrow \infty} D_{s_{j n}}\left(u_{j n}\right)+D_{s_{j N}}\left(v_{j N}\right)
$$

and

$$
0=-\lim _{n \rightarrow \infty} D_{s_{j N}}\left(u_{j N}\right)+\lim _{n \rightarrow \infty} D_{s_{j N}}\left(v_{j N}\right)
$$

We now conclude that the continuous function $u_{j}=v_{j}^{\prime} \mid\left(T_{j}-\beta^{*}\right)$ on $T_{j}$ satisfies: 
$u_{j} \mid\left(T_{j}-\beta\right)$ is harmonic,

$u_{j}\left|S_{j N}=v_{j N}^{\prime}\right| S_{j N}$,

$D_{s_{j n}}\left(u_{j n}-u_{j}\right) \rightarrow 0$ as $n \rightarrow \infty$,

$D_{s_{j n}}\left(u_{j n}\right) \rightarrow D_{T_{j}}\left(u_{j}\right)>0$ as $n \rightarrow \infty$,

${ }^{*} d u=0$ along $\gamma_{j}$,

$u_{j n}(z) \rightarrow \dot{u}_{j}(z)$ as $n \rightarrow \infty$ for $z \in T_{j}-\beta$, and the convergence is uniform on every compact subset of $T_{j}$.

We have shown that

$$
0<\lambda\left(F_{j}\right)=D_{T_{j}}\left(u_{j}\right)^{-1}<\infty .
$$

Suppose that $\bigcup_{k=0}^{2} \Gamma_{C j}(k)=0$ for all $j$. Then $F_{j} \subset F_{j+1}$ and $F=\bigcup F_{j}$. It follows easily that

$$
0<\lambda(F)=\lim \lambda\left(F_{j}\right)=\lim D_{T_{j}}\left(u_{j}\right)^{-1}<\infty .
$$

If there exist a $j(0) \geqslant 1$ and a $k(0)=0,1$ or 2 such that $\Gamma_{C j(0)}(k(0)) \neq 0$, then $\Gamma_{C j}(k(0)) \neq 0$ for all $j \geqslant j(0)$. Hence,

$$
\lambda(F) \geqslant \lambda\left(F_{j+1}\right) \geqslant \lambda\left(F_{j}\right)>0 .
$$

Thus, since the family $H$ of all curves in $S-(\beta \cup \gamma)$ which join $\alpha_{0}-\beta$ to $\alpha_{1}-\beta$ is a subset of $F, 0<\lim \lambda\left(F_{n}\right) \leqslant \lambda(F)<\infty$.

The opposite inequality is established as before.

Note, for $m \leqslant n$,

$$
D_{T_{m}}\left(u_{m}, u_{n}\right)=\lim _{j \rightarrow \infty} D_{S_{m j}}\left(u_{m j}, u_{n j}\right)=\lim _{j \rightarrow \infty} D_{S_{n j}}\left(u_{n j}\right)=D_{T_{n}}\left(u_{n}\right) \text {. }
$$

Hence, $D_{T_{m}}\left(u_{m}-u_{n}\right)<D_{T_{m}}\left(u_{m}\right)-D_{T_{n}}\left(u_{n}\right)$, and $D_{T_{m}}\left(u_{m}-u_{n}\right) \rightarrow 0$ as $m, n \rightarrow \infty$.

We conclude that there exists a continuous function $u$ on $S-\beta^{*}$ which is harmonic on $\vec{R}, 0$ on $\alpha_{0}, 1$ on $\alpha_{1}$, a constant on each component $\nu$ of $\alpha_{2}$ with the flux of $u$ along $\nu$ equal to 0 and ${ }^{*} d u=0$ along $\gamma$ such that $0<\lambda(F)=D_{\bar{R}}(u)^{-1}<\infty$.

7. Final result. It is also possible to assume that $\alpha_{0}, \alpha_{1}$ and $\alpha_{2}$ each contain perfect nowhere dense subsets of $\mathrm{Cl}(\partial \bar{R})$.

Suppose that the type II contour $\sigma_{1}$ of $\bar{R}$ intersects a perfect nowhere dense subset of $\alpha_{k}(k=0,1$ or 2$)$ and that as we traverse the component of $\partial S$ which contains $\sigma_{1}$ in the positive sense from $\sigma_{1}$ we traverse the type II contours $\sigma_{1}, \sigma_{2}, \cdots, \sigma_{n}$ of $\vec{R}$. We shall define the $M$-perfect nowhere dense subset of $\alpha_{k}$ which intersects $\sigma_{1}$.

For each $j=1, \cdots, n$ let $\delta_{j}$ denote the union of all perfect nowhere 
dense subsets of $\alpha_{k}$ on $\sigma_{j}$ and set $\delta_{n+1}=\delta_{1}$. If $\delta_{1} \cap \delta_{2} \neq 0$ (respectively $\delta_{1} \cap \delta_{n} \neq 0$ ) let $s(r)$ denote the largest integer such that $\delta_{j} \cap \delta_{j+1} \neq 0$ $\left(\delta_{n+2-j} \cap \delta_{n+1-j} \neq 0\right)$ for $j=1, \cdots, s-1 \quad(j=1, \cdots, r)$. Define the $M$-perfect nowhere dense subset $\delta$ of $\alpha_{k}$ which intersects $\sigma_{1}$ as follows:

$$
\begin{aligned}
& \delta=\delta_{1} \text { if } \delta_{1} \cap \delta_{2}=0 \text { and } \delta_{1} \cap \delta_{n}=0 \\
& \delta=\bigcup_{j=1}^{s} \delta_{j} \text { if } \delta_{1} \cap \delta_{2} \neq 0 \text { and } \delta_{1} \cap \delta_{n}=0 \\
& \delta=\bigcup_{j=0}^{r} \delta_{n-j+1} \text { if } \delta_{1} \cap \delta_{2}=0 \text { and } \delta_{1} \cap \delta_{n} \neq 0 ; \\
& \delta=\bigcup_{j=1}^{s} \delta_{j} \cup \bigcup_{j=1}^{r} \delta_{n-j+1} \text { if } \delta_{1} \cap \delta_{2} \neq 0 \text { and } \delta_{1} \cap \delta_{n} \neq 0 .
\end{aligned}
$$

If a type I contour $\sigma$ of $\bar{R}$ contains a perfect nowhere dense subset of $\alpha_{k}$ we define the $M$-perfect nowhere dense subset of $\alpha_{k}$ which intersects $\sigma$ to be the union of all perfect nowhere dense subsets of $\alpha_{k}$ on $\sigma$.

Clearly, two $M$-perfect nowhere dense subsets of $\alpha_{k}$ are either equal or disjoint.

Let $\tau_{k}$ and $\tau_{l}$ denote $M$-perfect nowhere dense subsets of $\alpha_{k}$ and $\alpha_{l}$, respectively, $l \neq k$ and $l, k=0,1$ or 2 . Suppose that $\tau_{k}$ and $\tau_{l}$ intersect the contour $\Sigma^{\prime}$ of $\bar{R}$. If $\Sigma^{\prime}$ is a type I contour of $\bar{R}$, take any point $p$ on $\gamma$ that is "between" $\tau_{k}$ and $\tau_{l}$, that is, take any point $p$ on $\gamma$ such that as we traverse $\Sigma^{\prime}$ in the positive (respectively negative) sense from $p$ we traverse a subset of $\tau_{k}$ (respectively $\tau_{l}$ ) before we traverse a subset of $\tau_{l}$ (respectively $\left.\tau_{k}\right)$. Set $\Sigma=\Sigma^{\prime}-\{p\}$ if $\Sigma^{\prime}$ is type I and $\Sigma=\Sigma^{\prime}$ otherwise.

There is a homeomorphism $H$ of $\Sigma$ onto $\{t: 0<t<1\}$. Assume that $H\left(\tau_{k}\right)$ is the Cantor set on $T_{a}=\{t: a \leqslant t \leqslant b\}$ (possibly minus one or both end points) and that $H\left(\tau_{l}\right)$ is the Cantor set on $\{t: c \leqslant t \leqslant d\}$ (possibly minus one or both end points), $0 \leqslant a<b \leqslant 1$ and $0 \leqslant c<d \leqslant 1$. Assume that $a<c$. We shall say that $\tau_{k}$ and $\tau_{l}$ intersect if $a<c<b$ and that $\tau_{k}$ and $\tau_{l}$ are disjoint if $b<c$. If $\tau_{k}$ and $\tau_{l}$ intersect, as we traverse $T_{a}$ in the positive sense from $a$ to $b$ we meet $\tau_{k}(1), \tau_{l}(1), \tau_{k}(2), \tau_{l}(2), \cdots, \tau_{l}(n)$, $\tau_{k}(n+1)$, where $\tau_{k}(j)$ is a subset of $\tau_{k}, \tau_{l}(j)$ is a subset of $\tau_{l}$ and $1 \leqslant n<\infty$. Call $n$ the number of times $\tau_{l}$ and $\tau_{k}$ intersect on $\Sigma$.

Note, if $\Sigma^{\prime}$ is a type I contour of $\bar{R}$ and $p_{1}$ and $p_{2}$ are any two points of $\gamma$ "between" $\tau_{k}$ and $\tau_{l}$, then the number of times $\tau_{k}$ and $\tau_{l}$ intersect on $\Sigma^{\prime}-\left\{p_{1}\right\}$ is equal to the number of times $\tau_{k}$ and $\tau_{l}$ intersect on $\Sigma^{\prime}-\left\{p_{2}\right\}$.

Let $\left\{\tau_{L}(j)\right\}_{j=1}^{J(L)}$ denote the $M$-perfect nowhere dense subsets of $\alpha_{L}$, $L=0,1$ and 2. Suppose that the subsets $\tau^{\prime}$ and $\tau^{\prime \prime}$ of $\tau_{k}(j)$ intersect a component $K$ of $\partial S$. We shall say that $\tau^{\prime}$ and $\tau^{\prime \prime}$ are separated by $A=$ $\bigcup_{L=0}^{2} \bigcup_{j=1}^{J(L)}\left(\tau_{L}(j)-\tau_{k}(j)\right)$ if as we traverse $K$ in both the positive and negative sense from $\tau^{\prime}$ we traverse subsets of $A$ before traversing $\tau^{\prime \prime}$.

For each $j=1, \cdots, J(k)$ decompose $\tau_{k}(j)$ into a finite number of subsets $\left\{\tau_{k}(j, s)\right\}_{s=1}^{S(j)}$ so that $\tau_{k}(j, s)$ and $A$ are disjoint and so that for $s \neq t$, 
$\tau_{k}(j, s)$ and $\tau_{k}(j, t)$ are separated by $A$. We shall mean when we say that " $\alpha_{k}$ contains a finite number of perfect nowhere dense subsets of $\mathrm{Cl}(\partial \bar{R})$ " that $\alpha_{k}$ is the finite union of the sets $\tau_{k}(j, s), s=1, \cdots, S(j)$ and $j=1, \cdots$, $J(k)$.

We now state our general result.

THEOREM 7. Let $S$ denote the compactification of $\bar{R}$ defined above. Let $\beta=S-\bar{R}$ and $\partial S=\beta \cup \partial \bar{R}$. Suppose that $\alpha_{0}, \alpha_{1}$ and $\alpha_{2}$ are closed subsets of $\partial S$ which satisfy (6.1), (6.2), (6.3) and

(7.1) $\alpha_{0}, \alpha_{1}$ and $\alpha_{2}$ are each composed of points of $\beta$, type I contours of $\bar{R}$ and a finite number of arcs and perfect nowhere dense sets on $\partial S$.

Set $\gamma=\partial S-\left(\alpha_{0} \cup \alpha_{1} \cup \alpha_{2}\right)$ and denote by $F$ the family of all curves in $S-\gamma$ which join $\alpha_{0}$ to $\alpha_{1}$. Let $\beta^{*}=\mathrm{Cl}(\partial \bar{R})-\left(\alpha_{0} \cup \alpha_{1} \cup \alpha_{2} \cup \partial \bar{R}\right)$. Then there exists a continuous function $u$ on $S-\beta^{*}$ which is harmonic on $\bar{R}$, 0 on $\alpha_{0}, 1$ on $\alpha_{1}$, a constant on each component $\nu_{j}$ of $\alpha_{2}$, with the flux of $u$ across $v_{j}$ equal to zero and ${ }^{*} d u=0$ along $\gamma$ such that $0<\lambda(F)=$ $D_{\bar{R}}(u)^{-1}<\infty$.

The proof of this result is similar to that of Theorem 6 and will be omitted.

\section{BIBLIOGRAPHY}

1. L. V. Ahlfors, Complex analysis: An introduction to the theory of analytic functions of one complex variable, 2nd ed., McGraw-Hill, New York, 1966. MR 32 \#5844.

2. L. V. Ahlfors and L. Sario, Riemann surfaces, Princeton Math. Ser., no 26, Princeton Univ. Press, Princeton, N. J., 1960. MR 22 \#5729.

3. J. A. Jenkins, Univalent functions and conformal mapping, 2nd ed., Springer-Verlag, Berlin and New York, 1965.

4. - Lecture notes at Washington University, 1968-1969.

5. A. Marden and B. Rodin, Extremal and conjugate extremal distance on open Riemann surfaces with applications to circular-radial slit mappings, Acta Math. 115 (1966), 237-269. MR 34 \#2862.

6. C. D. Minda, Extremal length and harmonic functions on Riemann surfaces, Trans. Amer. Math. Soc. 171 (1972), 1-22.

7. Z. Nehari, Conformal mapping, McGraw-Hill, New York, 1952. MR 13, 640.

8. B. Rodin and L. Sario, Principal functions, Van Nostrand, Princeton N. J., 1968. MR 37 \#5378.

9. L. Sario and M. Nakai, Classification theory of Riemann surfaces, Die Grundlehren der math. Wissenschaften, Band 164, Springer-Verlag, Berlin and New York, 1970.

MR 41 \#8660.

10. L. Sario and K. Oikawa, Capacity functions, Die Grundlehren der math. Wissenschaften, Band 149, Springer-Verlag, Berlin and New York; 1969. MR 40 \#7441.

11. G. Springer, Introduction to Riemann surfaces, Addison-Wesley, Reading, Mass., 1957. MR 19, 1169.

DEPARTMENT OF MATHEMATICS, EMORY UNIVERSITY, ATLANTA, GEORGIA 30322

Current address: Department of Mathematics, Georgia Institute of Technology, Atlanta, Georgia 30332 